\title{
Experimental and theoretical analysis of large particle-wall collision with different metal plates
}

\author{
Xueyu Chen ${ }^{1} \cdot$ Langyu $\mathrm{Ji}^{1} \cdot \mathrm{Yi} \mathrm{Li}^{1}$
}

Received: 19 February 2021 / Accepted: 15 January 2022 / Published online: 10 February 2022

(c) The Author(s) 2022

\begin{abstract}
The coefficients of restitution are important parameters in the study of particle-wall collision, which is widely used in discrete element modeling in solid-liquid multiphase systems. To improve the accuracy of numerical simulations, correspondingly exact particle-wall collision parameters are needed. The current models used in numerical simulation, however, are mostly suitable for solid-gas systems, in which collision models used to be micro-sized. Millimeter-size particle-wall collision models in wet experimental conditions and research on the influence of plate materials were lacking. In this study, we designed an experimental system focused on the different materials of the particles and collision plates and the dry or wet experimental environment to discuss the change of restitution coefficients in various conditions. We selected millimeter-sized iron 45 particles and glass particles, which represented plastic material and hard brittle material, respectively. We also selected three industrial metals, HT250 gray cast iron, 6061 aluminum alloy, and 316 stainless steel, which are commonly used in hydraulic machinery, for the collision plates. Experiments were performed to measure the incident and rebound velocities of the particle at various impact angles. In this paper, we have provided and compared the results of restitution coefficients of velocity and angle varying with the approach angle. In the processing of the experiment, we used the control variate method and applied an ultra-depth of field three-dimensional microscope to scan the surface of the collision plate. In the analysis of the experimental results, single particle-wall collision was seen as a non-smooth dynamical system to discuss the specific phenomenon. Finally, we obtained the relationship between the restitution of normal velocity and elastic elements of materials and explained the relationship of stick-slip motion with the approach angle and velocity.
\end{abstract}

Keywords Particle $\cdot$ Wall collision $\cdot$ Non-smooth dynamics $\cdot$ Restitution coefficient $\cdot$ Stick-slip motion

\section{Introduction}

Collision is a basic research object in the physical sciences, and it also plays a key role in industrial processes like filtration, agglomeration, spray coating, and multiphase transmission. For the simplification of study, the typical collision model, like a particle shot toward a random object, has been simplified to a specific plate. This kind of particle-wall collision model has provided a theoretical foundation that influences the accuracy of numerical simulations that involve

Technical Editor: Erick Franklin.

Yi Li

liyi@zstu.edu.cn

1 State-Province Joint Engineering Lab of Fluid Transmission System Technology, Zhejiang Sci-Tech University, Hangzhou 310018, People's Republic of China collisions, especially in the simulation of multiphase transport processes. Therefore, it is necessary to study the mechanism of the particle-wall collision and build a reliable collision model.

The first classical analysis was established by Hertz [1], who assumed perfectly elastic collisions in a normal direction and provided definitions of coefficients of restitution. Researchers then focused on establishing models in dry surface conditions. Johnson [2] proposed the JKR theory [3], which takes the particle adhesion force in the inelastic collisions into account. Furthermore, Maw, Barber, and Fawcett [4] extended the Hertz theory to oblique collision. Sommerfeld [5] studied the small particle-wall collision model and the relationships between the coefficient of restitution, surface roughness, rotating angular velocity of particles, and the coefficient of friction. A significant achievement was proposed by Grant et al. [6], who put forward the first suitable particle-wall collision model for gas-solid two-phase flow 
based on an experiment in 1975. They thought the coefficient of restitution of particle-wall collisions was a function of particle impact angle, and this model has continued to be used widely in numerical simulations in recent years. These researchers have explained how to build a particle-wall collision model, as well as how to build the mathematical model through the simplified collision experiments. These researches established the foundation of the study on particle-wall collision, which mostly based on Newton's impact hypothesis [7]. In this theory, the restitution coefficients, like restitution coefficient of velocity $\left(e_{v}\right)$ and restitution coefficient of angle $\left(e_{\beta}\right)$, were mostly focused on. The restitution coefficients were defined by the ratios of particles' motion parameters, such as the velocities of the particle and the angle between the angle with the plate, before and after the collision, and were used to describe the influence of the particle-wall collision. According to these researchers, the most influent elements among several elements in the particle-wall collision process used to be the impact velocity and impact angle.

In the following research, collision models in different experimental conditions were designed by various researchers. In 1987, Tabakofft [8] carried out a particle-wall collision experiment in gas-solid two-phase flow, which took wall materials in account. They used the method of laser measurement to conclude collision models of particles with 410 stainless steel, 2024 aluminum alloy, and 6a1-4 $\mathrm{v}$ titanium alloy. At almost the same time, the theoretical studies of collision were developed. Davis [9] developed the elastohydrodynamic lubrication theory, which was based on the particle-particle vertical collisions experiment in a viscous fluid. Davis put forward the first standard to estimate whether or not the particle would rebound in the liquid. Based on Davis's theory, Kantak [10] furthered studies on the particle-wall collision and particle-particle collision of the wet surface and analyzed particle motion by recording the change of classic coefficients of restitution along with the impact angle and the impact velocity. Although these researchers used large particles in the collision experiments, they were focused mostly on head-on collision.

Recently, the research trend has turned to experimental and numerical studies on particle collision with the thin liquid film or under humid condition. Mueller [11] has conducted experiments on the oblique impact of three types of wet granules - that is, the dominant elastic $\boldsymbol{\gamma}-\mathrm{Al}_{2} \mathrm{O}_{3}$, the elastic-plastic zeolite $4 \mathrm{~A}$, and the dominant plastic sodium benzoate. They discussed how the properties and moisture content of particles influence the restitution. The particle-particle collision in the viscous medium was studied by Yang [12]. Stocchino [13] investigated the particle collision rule in the Newton fluid and non-Newton fluid. Joseph [14] studied the particle-wall collisions immersed in the liquid, which had different viscosity, and concluded that the change of the coefficient of restitution was determined mainly by the Stokes number. Gollwitzer et al. [15] examined the particle-wall collisions on wet surface and analyzed the energy dissipation in the process. They divided the energy dissipation into energy loss caused by collisions and the loss caused by liquid layer. Givehchi and Tan [16] investigated the behavior of liquid bridge force and elastic-plastic impaction in a collision under humid condition through the experiment and theoretical analysis. The particle-flow behavior in dry and humid multiple-spout fluidized beds was studied trough both experimental and simulation methods by Tang [17]. In 2018, Li [18] established a modified formula to calculate the normal restitution coefficient of particles in humid environment and studied the relationship between dry and humid normal restitution coefficients through experiment and theory. These collision models, however, were suitable mostly for small particles or light particles, although they had built a particle-wall collision model in obliquity collision.

The most common collision models used in recent simulations, including the Grant model, are almost all based on experiments conducted in dry surface condition. To increase the precision in simulation about multiphase flow, it is of great importance to establish a reliable collision model for specific condition. Hence, a lack of reliable collision about large particles with a wet hitting surface is a key point of this study. We focused on particle-wall collisions with two kinds of large-diameter particles using different materials in viscous fluid against three different types of plate materials.

In this study, we designed particle-wall collision experimental equipment and installed a high-speed camera to capture the collision instant phenomenon. Then we obtained the motion data of particles before and after rebounding by computer vision technology. In addition, a classic model of particle-wall collision was established by giving four kinds of coefficients of restitution. For studying the mechanism of particle-wall collision, we established orthogonal coordinates by the parallel and vertical directions of the collision plane. And the following discussion was explained in these two collision directions. We analyzed how the impact angle influenced the restitution and also provided a series of results of experiments on the impact of the velocity's effect. We used a micro-photo of the plates after collision to explain changes to the coefficients.

\section{Materials and experimental methods}

\subsection{Granule sample and target plate}

For usual use in the abrasion experiment, we selected two kinds of particles: 45 carbon steel and glass. As shown in Table 1, the elongation of 45 carbon steel was $40 \%$ and the 
elongation of glass was $1.2 \%$. Because the elongation of the plastic material was less than 5\%, 45 carbon steel represented a tough plastic material, and the glass represented a brittle material. The main properties of these granules are shown in Table 1.

Figure 1 shows the measurement of 100 particle samples of the two materials. The statistical histogram shows a

Table 1 Material characteristics of particles used in the experiments

\begin{tabular}{llll}
\hline Material & Elongation (\%) & $\begin{array}{l}\text { Young's modu- } \\
\text { lus }(\mathrm{GPa})\end{array}$ & Poisson's ratio \\
\hline Iron 45 & 40 & 210 & 0.269 \\
Glass & 1.2 & 46.2 & 0.245 \\
\hline
\end{tabular}

quasi-Gaussian distribution centered on $7 \mathrm{~mm}$, which represented the diameter of the particles, which was regarded as having the same shape. The diameters of both kinds of particles were $7 \mathrm{~mm}$.

We made plates using three kinds of materials for this experiment, as shown in Fig. 2, and all of them had the same shape. The plates were made of three different materials: 316 stainless steel plate, 6061 aluminum alloy plate, and HT250 gray cast iron. These materials are the most widely used materials in the flow component of fluid machinery. The micro-topography of the surfaces of plates also is shown in the figure. Considering the coefficient of restitution would rise with an increase in the ratio between the wall thickness and the diameter of particle $(D / b)$, the coefficient tended to
Fig. $17 \mathrm{~mm}$ particles used in the experiment
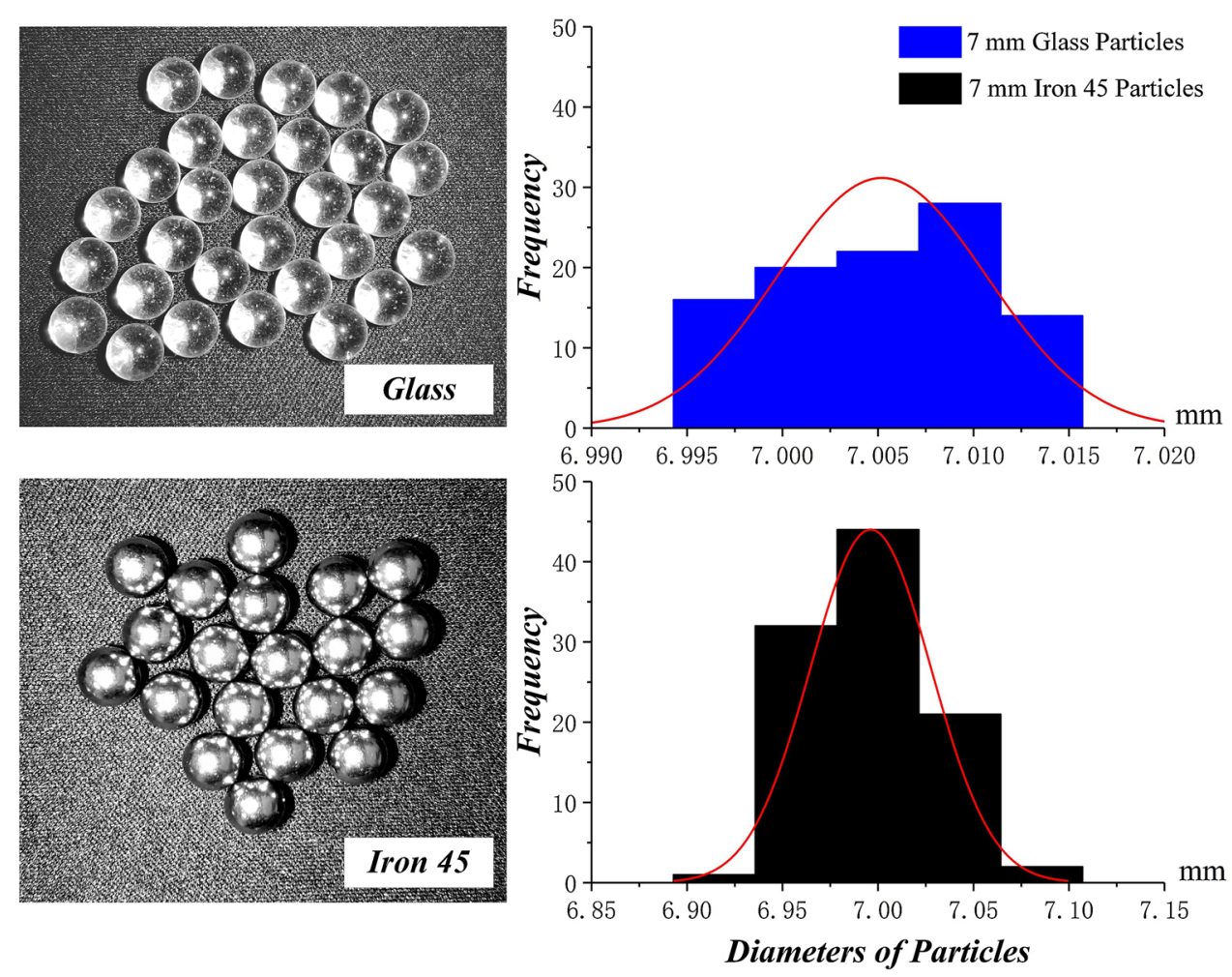

Fig. 2 Plates made of different materials used in the experiment
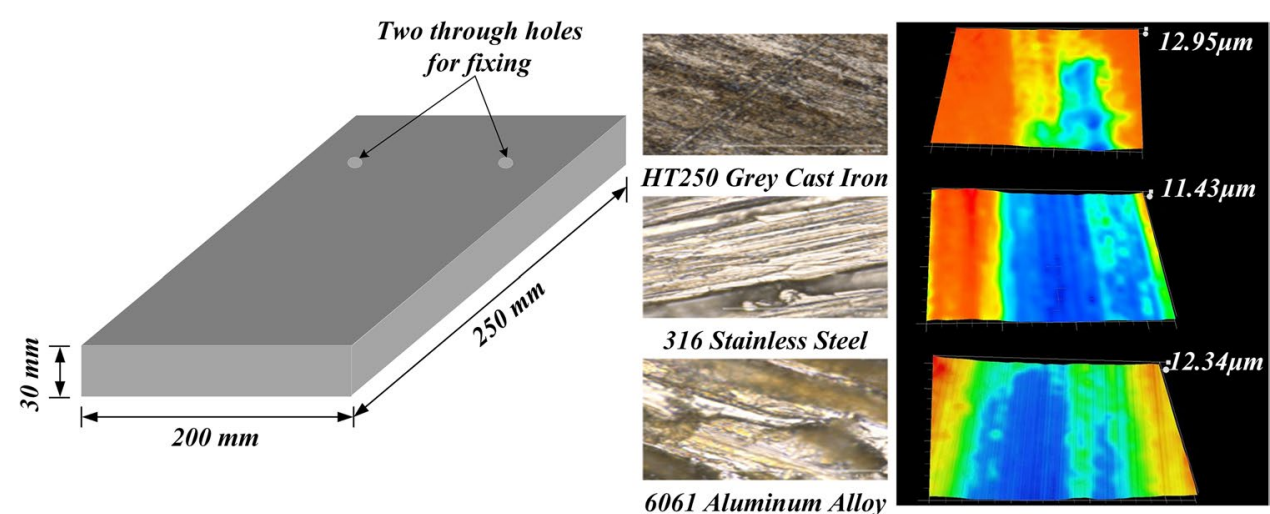
be stable until $D / b>4$ [19]. The thickness of the target plate was $30 \mathrm{~mm}$ to ensure that the results of the experiments were convincing.

The main characteristics of the materials used in our experiments are given in Table 2. Three plates had the same machine accuracy (Ra1.6). In addition, we obtained the roughness of the surfaces using the KEYENCE VHX-200 digital microscope. By measuring the maximum height different of the surfaces, which represented the rough depth Ry, we considered the three plates to have the same surface roughness. As shown in Fig. 2, although three plates had the similar surface roughness, the surface micro-topography were different.

As shown in Fig. 3, the device for the particle-wall collisions experiment was composed of a particle release device,

Table 2 Material characteristics of plates used in the experiments

\begin{tabular}{llll}
\hline Material & $\begin{array}{l}\text { Roughness } \\
(\mathrm{Ry})(\mu \mathrm{m})\end{array}$ & $\begin{array}{l}\text { Young's } \\
\text { modulus } \\
(\mathrm{GPa})\end{array}$ & Poisson's ratio \\
\hline 316 stainless steel & 12.95 & 206 & 0.3 \\
6061 aluminum alloy & 11.43 & 71 & 0.31 \\
HT250 gray cast iron & 12.34 & 135 & 0.3 \\
\hline
\end{tabular}

tank, angle-adjustable specimen rack, and a high-speed CCD camera. The core part of this equipment was the angleadjustable specimen rack, which consisted of a tilted plane and a support. A bolt was used to connect these two parts, which kept the inclined plane at a fixed angle, and the specimen rack was adjusted to a slope between 0 and 75 degrees. We adapted target plates using three different materials to fit this rack via bolts. When the angle-adjustable specimen holder was put in place, the release device dropped a granule in specific velocity, which was determined by the height of the release of the particle. A graduated metal rod was used to determine the height of release. Each drop was repeated 15 times to ensure the accuracy of the data.

\subsection{The experiment design}

As shown in Fig. 4, obvious changes occurred in the motion of the particle after the collision. In this study, we used Newton's restitution coefficients. These restitution coefficients are based on Newton's impact hypothesis, which described the influence of particle-wall collision according to the changes in the velocity and angle of the particle. We considered four core coefficients of the particle-wall collisions: $e_{v}, e_{n}, e_{t}$, and $e_{\beta}$, which represented the restitution coefficient of velocity, the restitution coefficient of normal velocity, the

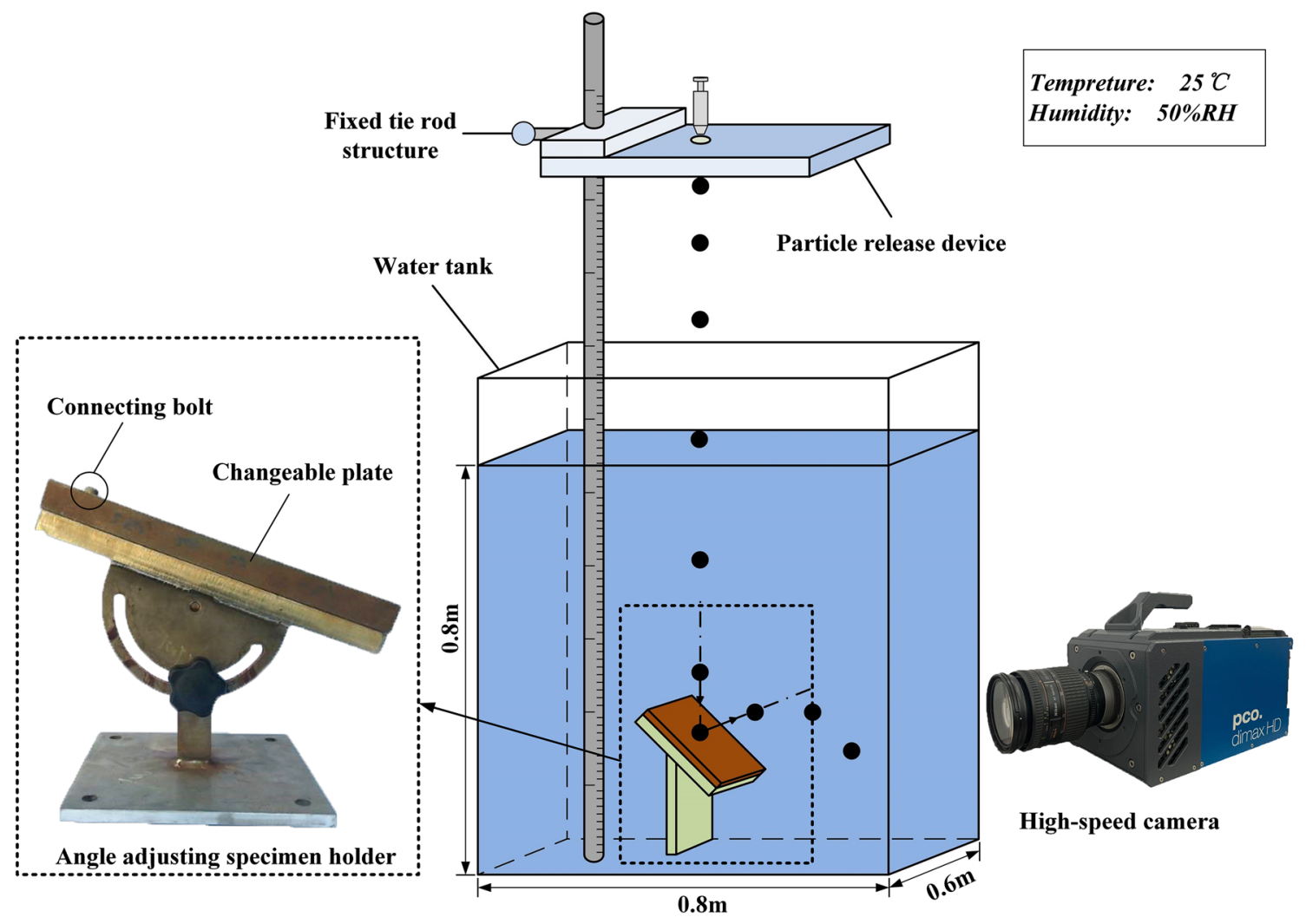

Fig. 3 Experimental system and the specimen materials used in the experiment 
Fig. 4 Schematic diagram of the particle-wall and motion parameters in the process

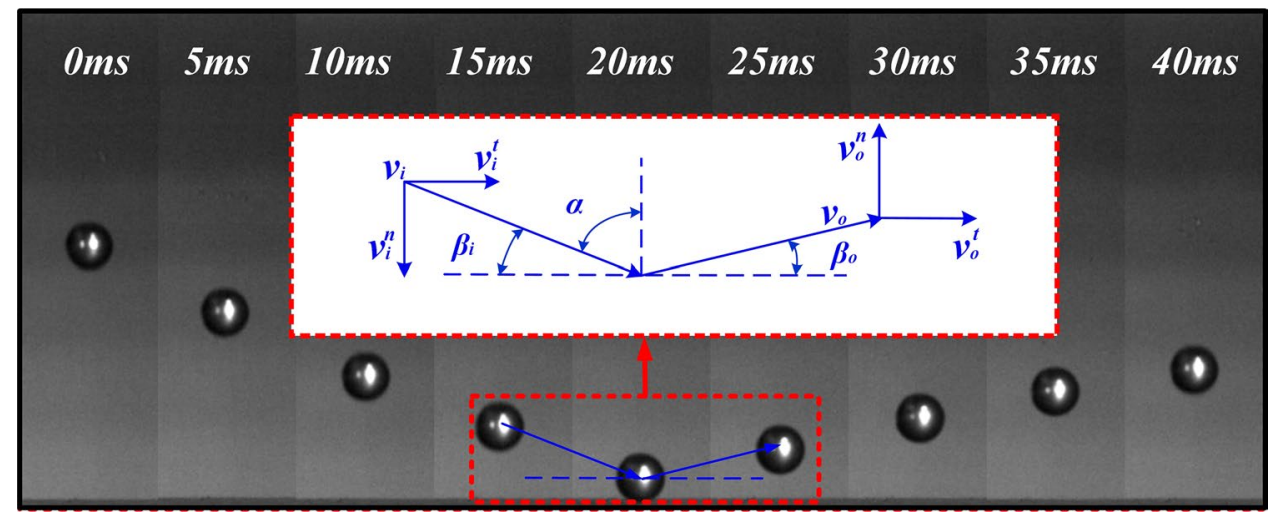

restitution coefficient of tangential velocity, and the restitution coefficient of the angle, respectively. These coefficients described the process of the particle-wall collision using several kinematics parameters, including the velocity of the particle $\left(v_{i}\right.$ and $\left.v_{o}\right)$, and the impact and the rebound angle $\left(\beta_{i}\right.$ and $\beta_{o}$ ), as shown in Fig. 4. Additionally, $v^{n}$ and $v^{t}$ are the normal and tangential component of the velocity.

In this study, we selected $e_{\nu}$ and $e_{\beta}$ to be the result indexes. The calculation expressions are as follows:

$e_{v}=\frac{v_{o}}{v_{i}}$, and

$e_{\beta}=\frac{\beta_{o}}{\beta_{\mathrm{i}}}$.

The restitution coefficient of velocity $e_{v}$ and the restitution of angle $e_{\beta}$ described the change of the motion of particles before and after the impact through the ratio of velocity and angle. From Fig. 4, we know that the restitution coefficient of velocity $e_{v}$ is the ratio between the rebound velocity and the approach velocity, and the angle coefficient of restitution $e_{\beta}$ is the ratio between the rebound angle and the impact angle.

We completed two series of experiments. The first experiment was based on changes in the impact angle, and the second experiment was based on the impact velocity.

To build the particle-wall collision model and simplify the experiments, we considered more experimental factors in the first series of experiments. The first experimental matrix is provided in Table 3.

This paper considered four controllable factors ( $A$ : Angle of the plate, $B$ : Material of the plate, $C$ : Material of the particle, and $D$ : Experimental environment). The different materials of the plate and the particle represented the influence of the material characteristics in the collision, and the dry and wet experimental conditions represented the influence of the fluid properties. The drop height of the particle in dry condition was $0.8 \mathrm{~m}$. In the wet collision, the depth of water was
$0.8 \mathrm{~m}$, we have tried different drop heights and compared the impact velocities of the particles in the liquid. By this way, we made sure the impact velocities of the particles in dry and wet collision the same.

Then, we conducted a series of experiments aimed at researching the influence of the impact velocity following the guidance of the control variable method, which meant that only the impact velocity changed in the experiment and that other factors remained the same. We selected a deformable aluminum alloy plate as the specimen and selected an impact angle of 50 degrees. The impact velocities ranged from 1.0 to $5.0 \mathrm{~m} / \mathrm{s}$, and 30 drop positions were selected to control the different velocities. The impact velocity was controlled by the release height of the particle, which ranged from 0.4 to $1.6 \mathrm{~m}$. The height was determined by the graduation of the particle release device, and the drop height increased $5 \mathrm{~cm}$ at a time. The design of the second series of the experiment is provided in Table 4.

\subsection{Data acquisition procedure}

We filmed the particle-wall collision process using a CCD camera, whose exposure time was set as $0.5 \mathrm{~ms}$. For image processing, we used the frame differential method [20] to obtain the position coordinates of the particle and the movement angle between the particle and the plate (including the impact angle and rebound angle). The frame differential method is a way to find differences in two adjacent pictures. The first step in the operation was to recognize the particles in the pictures and then to calculate the distance of particles movement. Three photographs before and after the collision were imported in to MATLAB software. The edge and center coordinates of the particles could be identified using the Imfindcircles function [21]. We used the sphere edge identification procedure to identify the location of the ball and obtain the center coordinate. The center position was described by the pixel coordinate values, which we obtained according to the resolution of the picture. We obtained the 
Table 3 Experimental matrix based on impact angles

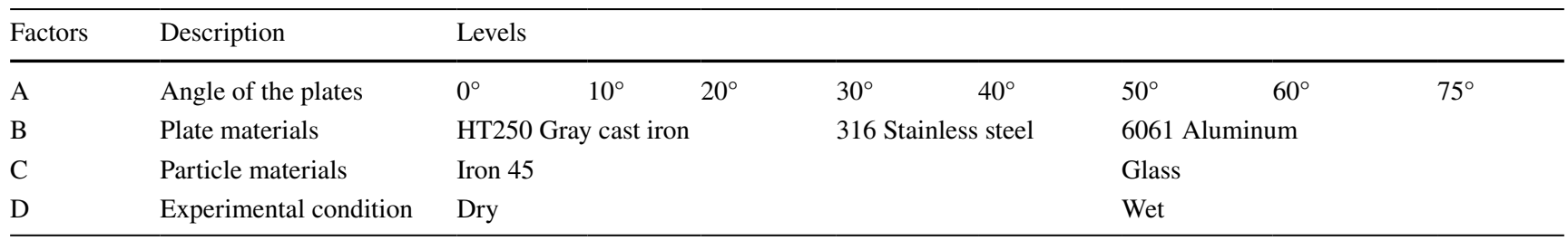

Table 4 Experimental matrix based on the impact velocity

\begin{tabular}{lll}
\hline Factors & Factors & Levels \\
\hline A & Release height & $0.4-1.6 \mathrm{~m}$ \\
B & Impact velocity of particles & $1-5 \mathrm{~m} / \mathrm{s}$ \\
C & Materials of the particle and plate & Iron 45 and \\
& & 6061 alu- \\
& & minum alloy \\
D & Impact angle of particles & $50^{\circ}$ \\
\hline
\end{tabular}

particle velocity and the angle before and after the collision using the conversion of the pixel coordinate.

As shown in Fig. 5, an assumption was made that the granule affected the plate vertically to ensure that the impact angle of the particles and plates $\left(\beta_{i}\right)$ could be represented by the inclination angle of the specimen rack $(\alpha)$. The relationship of the impact angle of the dip angle can be shown as follows:

$\beta_{i}=90^{\circ}-\alpha$

where the impact angle $\beta_{i}$ means the angle between the vertical line and the inclined plane. Because of the relatively large density of the particle, it would fall along a vertical line.

According to Davis et al. [9], the farthest distance of approach between particle and plate when an elasto-hydrodynamic collision occurs is calculated as follows:

$h_{e h}=\left(4 \mu K v^{n} R^{3 / 2}\right)^{2 / 5}$,

where $\mu$ is the dynamic viscosity of the liquid, $v_{i}^{n}$ is the normal component of the impact velocity $v_{i}$, and $R$ is the radius of the particle. And $K$ is defined as follows:

$K=\left(\frac{1-v_{1}^{2}}{E_{1}}+\frac{1-v_{2}^{2}}{E_{2}}\right)$,

where $E_{i}$ represents Young's modulus and $\nu_{i}$ represents Poisson's ratio for the particle $(i=1)$ and plane $(i=2)$, respectively. The Poisson's ratios and Young's modules of the particles and the plates are given in Table 1.

In the elasto-hydrodynamic collision, the fluid would act like lubrication between the particles and the plates, which

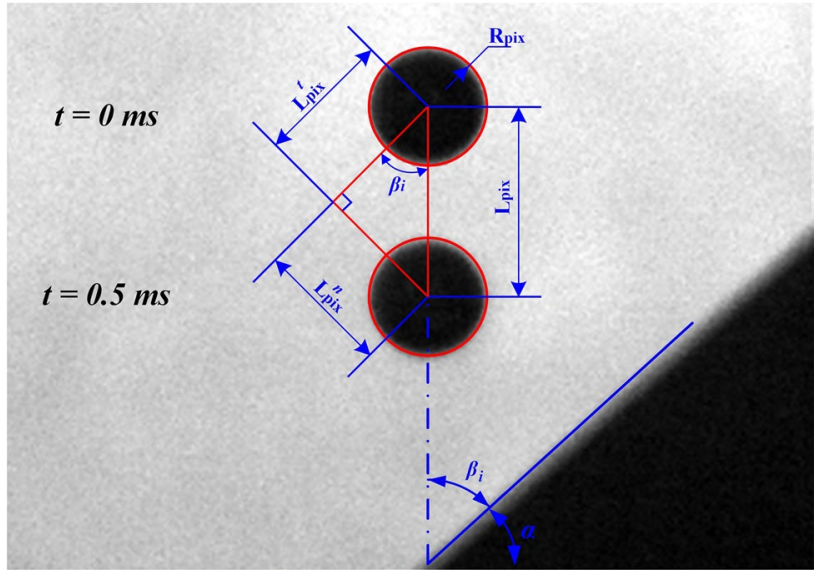

Fig. 5 Identification of particle boundary in Matlab software

means the trajectory of the particle may not keep straight when it was closed to the surface of the plate. The specific drop height for wet experimental condition was larger than $80 \mathrm{~cm}$, which was much larger than the $h_{e h}$. Therefore, the particle would impact the plate straightly, and the liquid would not act like lubrication to make trajectory of the particle bend.

As shown in Fig. 5, the velocity of the particle can be calculated by the pixel distance of two adjacent pictures. The calculation of the impact velocity of the particles is as follows:

$v_{i}=\frac{L_{\mathrm{pix}}}{\Delta t} \frac{R}{R_{\mathrm{pix}}}$,

where $v_{i}$ is the impact velocity, which can be calculated by the drop distance and the time interval between two frames; $L_{\text {pix }}$ indicates the pixel distance between the two adjacent pictures; and $\Delta t$ is equal to the interval of time between the two adjacent pictures. The pixel distance needs to be multiplied by a ratio, which is equal to the ratio of the real radius of particle $R$ and the pixel radius of particle $R_{\text {pix }}$.

The particles would deform badly when the material reached its elastic yield limit. The elastic yield limit was attained only for incident velocities bigger than $v_{\varepsilon}$, which is a limiting velocity given by Jonson [22]: 
$v_{\varepsilon}=\left(\frac{2 \pi}{K}\right)^{2}\left(\frac{2}{5 \rho}\right)^{\frac{1}{2}} \varepsilon^{\frac{5}{2}}$,

where $\varepsilon$ is the elastic parameters given in formula (14), $K$ is the parameter given in formula (5). This formula was used to determine whether the particle attained at the elastic yield and the material was irreversibly deformed. According to the value of parameters given in Table 5, the limiting elastic velocity was $21.7 \mathrm{~m} / \mathrm{s}$ for the iron particle, and the impact velocities of the particles in the experiment ranged from 1 to $5 \mathrm{~m} / \mathrm{s}$. Therefore, the impact velocities were controlled under this limiting velocity to maintain that the deformation of the particle was not that huge and reversible, for the particles could be seen as circles in the MATLAB software.

According to the same principle, the calculation formula of normal and tangential impact velocity components are as follows:

$v_{i}^{n}=\frac{L_{\mathrm{pix}}^{n} R / R_{\mathrm{pix}}}{\Delta t}, L_{\mathrm{pix}}^{n}=L_{\mathrm{pix}} \sin \beta_{i}$, and

$v_{i}^{t}=\frac{L_{\mathrm{pix}}^{t} R / R_{\mathrm{pix}}}{\Delta t}, L_{\mathrm{pix}}^{t}=L_{\mathrm{pix}} \cos \beta_{i}$,

where $v_{i}^{n}$ is the normal impact velocity and $v_{i}^{t}$ is the tangential impact velocity, and $\beta_{i}$ is the impact angle.

The rebound angle is obtained as follows:

$\beta_{o}=\left(90^{\circ}-\beta_{i}\right)-\arctan \frac{\left(x_{2}-x_{1}\right)}{\left(y_{2}-y_{1}\right)}$,

where $x_{1}, x_{2}, y_{1}$, and $y_{2}$ are the pixel horizontal and vertical coordinates of the two adjacent pictures. Similarly, we used the same method to obtain the tangential impact velocity, the normal rebound velocity, and the tangential rebound velocity.

\section{Results}

\subsection{Experimental results around the impact angles}

To study the influence of the impact angle, we conducted a collision experiment using several plate angles for iron and glass particles with three different types of plate materials. Each experiment was carried out in two experimental environments - that is, drying and immersion. We studied the particle-wall collisions with a large particle diameter of $7 \mathrm{~mm}$. The average value was calculated based on 15 collision tests, and then we used the Grubbs test at a confidence rate of 0.95 (the significance level $\alpha=0.05$ ) to eliminate the accidental error. The restitution coefficient curves of HT250 gray cast iron, 6061 aluminum alloy, and 316 stainless steel
Table 5 Elasticity parameter in dry and wet experimental conditions

\begin{tabular}{llll}
\hline Particle & Plate & $\varepsilon_{\text {dry }}$ & $\varepsilon_{\text {wet }}$ \\
\hline Iron 45 & HT250 Gray cast iron & $7.943 \times 10^{-9}$ & $1.905 \times 10^{-7}$ \\
& 6061 Aluminum & $1.221 \times 10^{-8}$ & $2.927 \times 10^{-7}$ \\
& 316 Stainless steel & $6.825 \times 10^{-9}$ & $1.508 \times 10^{-7}$ \\
Glass & HT250 Gray cast iron & $1.804 \times 10^{-8}$ & $3.144 \times 10^{-7}$ \\
& 6061 Aluminum & $2.927 \times 10^{-8}$ & $3.839 \times 10^{-7}$ \\
& 316 Stainless steel & $1.649 \times 10^{-8}$ & $2.874 \times 10^{-7}$ \\
\hline
\end{tabular}

with two kinds of particles were as follows, and the results of the experiments in dry and wet environment are shown in Fig. 6.

Figure 6 is a constitution diagram that shows the particle-wall collisions curves of two key elements obtained directly by the experimental results. Note that $e_{v}$ and $e_{\beta}$ are the ratio of the results obtained from the experiment and are used to represent the change in the motion of the particles directly caused by the collision. The results showed the changes trending in the coefficient of the restitution in the velocity and angle of iron particles and glass particles before and after the collision along with the impact angles from $10^{\circ}$ to $90^{\circ}$, which were controlled by the oblique angles of the plate. According to the line graph, we could see that all four curves changed along with the impact angle from $10^{\circ}$ to $90^{\circ}$, which proved the validity of the results of the orthogonal experiment. The approaching angle could be a significant factor in the collision process. Figure $6 a, b$ represent the coefficient of velocity and angle of iron particles with different wall materials and at different angles, respectively. In Fig. 6a, the restitution coefficients of velocity decreased along with the increase of the impact angle. In contrast, the coefficients of the angle increased with the increase in the impact angle, as shown in Fig. 6b. In addition, the velocity and angle coefficients for the HT250 gray cast iron were bigger than the coefficients for 316 stainless steel, and the coefficients for 6061 aluminum alloy were the smallest. This relationship between the values of the coefficients for the different plate materials was the same as the values of the hardness of the corresponding plate materials. Moreover, Fig. 6c, $\mathrm{d}$ represented the coefficient of velocity and angle for the glass particles, respectively. There was a slight downward trend in the velocity curves when using the glass particles shown in Fig. 6c, and the velocity coefficients seemed to be dependent on the impact angles of the glass particles.

Along with an increase in the impact angle, the curves of the restitution coefficient of the velocity tended to decrease for both particles. Figure 6 a shows a very marked decreasing trend of the values of $e_{v}$, whereas Fig. $6 \mathrm{~b}$ shows the opposite trendthat is, the larger the impact angle, the smaller the value of $e_{\beta}$. The tendency for the glass particle was much slighter in 

of velocity and angle vary with approach angles: a $e_{v}$ of iron 45 particle; $\mathbf{b} e_{\beta}$ of iron 45 particle; $\mathbf{c} e_{v}$ of glass particle; and $\mathbf{d} e_{\beta}$ of glass particle
Fig. 6 Restitution coefficients

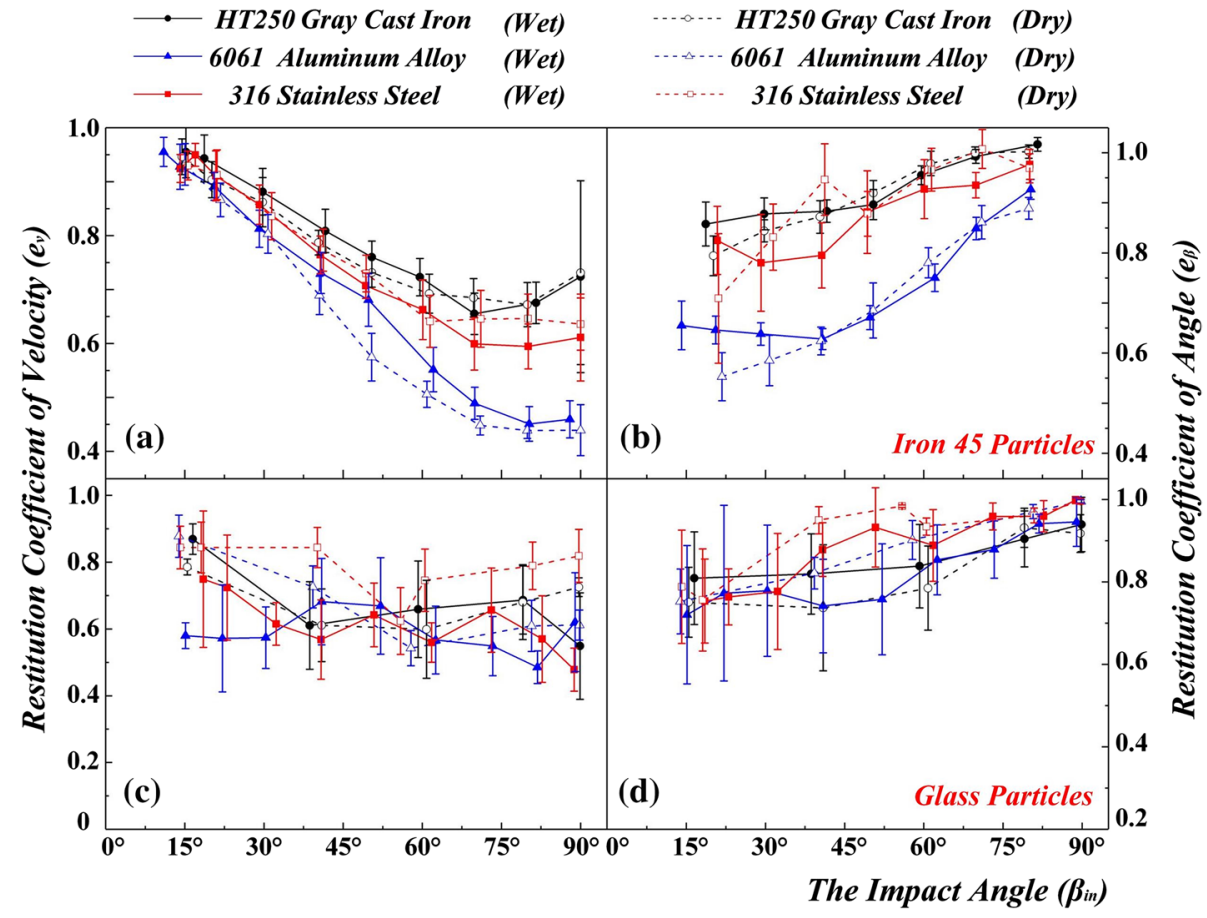

Fig. 6c, d, and the curves tended to be flat along with a bigger impact angle. Although the curves of the restitution coefficient of the angle increased as the impact angle increased for both particle materials, the curves of the glass particles did not follow obvious trends. This difference revealed that the deflection of glass particles was much less than the iron particles when the impact angle remained the same.

Meanwhile, there were slight differences between the different experimental environments. As shown in the comparison, the results of glass particles were not obvious, but the trends still could be summarized. In Fig. 6a, b, we found that for iron particles, the curves of the experiment in the dry environment were almost all below the curves of the wet experiments, which meant that the coefficients were bigger in the wet environment for large iron particles. This phenomenon switched in Fig. 6c, d, in which the values of the restitution coefficient from the dry environmental condition were almost all larger than the values of the wet surface condition. This phenomenon may have been caused by the different mass of the two kinds of particle materials, which meant that the iron had a much larger density than the glass, and the same uplift had a greater influence on the glass particles' motion.

An obvious phenomenon shown in Fig. 6 is the distinction for the different plate materials. The curves of HT250 gray cast iron were higher than the curves of the 316 stainless steel, and below the curves of these two plate materials were the curves of the 6061 aluminum alloy. This phenomenon may have been the result of the hardness of the different materials, and further theoretical research is available in the rest of this paper. Another interesting phenomenon is a twisting in the region where the impact angle was between 20 and 45 degrees. In this range, the variation trend of the restitution coefficient of the angle was not pronounced.

\subsection{Experimental results around the impact velocity}

To research how the influence of the impact velocity on the dependence of the stick-slip motion, another series of experiments were done. In regard to the discussion under the condition of the elastic stage, the particle velocity should be less than the limiting elastic velocity.

Figure 7 shows the relationship between the restitution coefficient and impact velocity. As we can see from Fig. 7, with the increase of the impact velocity, the velocity coefficient of restitution gradually decreased and finally tended to be stable, while the angle coefficient of the restitution decreased clearly with the increase of the impact velocity. This decrease meant that the lager the impact velocity was, the smaller rebound angle was under the same approach angle.

Johnson et al. [2] took data from Goldsmith for steel spheres affecting brass, bronze, and lead plates and found that the coefficient of restitution was proportional to a quarter power of velocity, which expresses the coefficient of restitution in the presence of plastic deformation by a relation of the following form:

$e=\mathrm{d} v_{i}^{-n}$ 
where $n=1 / 4$ and $\mathrm{d}$ is a constant that depends on material properties such as elastic moduli, Poisson's ratios, dynamic yield strengths, equivalent mass, and equivalent radius. Under this guidance, we fitted the solid curves in Fig. 7 using this function model in the allometric law.

Under an analysis based on theoretical mechanics and elastic mechanics, this decrease could be a little unusual. According to the generalized Hooke's law, the relationship between the stress and strain of the particles depends on the nature of the material in elastic state. Therefore, the coefficient of restitution of the angle and velocity should remain almost unchanged as the impact velocity increased in the condition of the elastic stage. The slight decline in the velocity coefficient of restitution, however, might be attributed to system error. The decrease in the angle coefficient was too obvious to be ignored, which meant that the rebound angle became smaller when the impact velocity became lager in the same approach angle. Such a decreasing trend in the angle coefficient of restitution has been discovered by many researchers.

\section{Discussion}

In the dynamical model of the single particle-wall collision, the collision plate can be seen as the only constraint in the motion, and a single particle would turn in different directions after the collision [23]. Therefore, the single particle wall can be taken as a non-smooth dynamical system.

\subsection{The grazing impact}

Discussions of the theoretical research about non-smooth dynamical systems focus on two specific motions: grazing impact and stick-slip bifurcation. The phenomenon of grazing impact refers to the uncertainty of motion when the

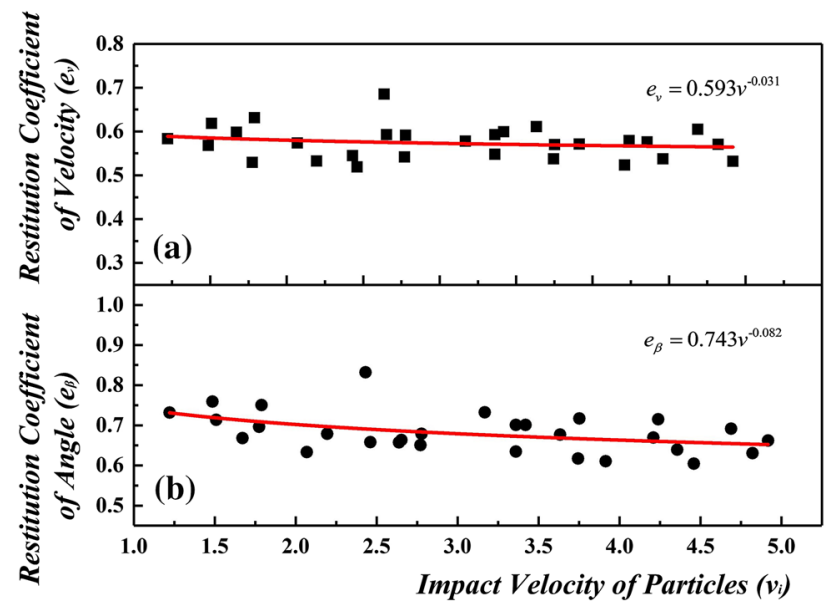

trajectory of a particle is in contact with the collision surface at zero velocity in the phase space [24]. The trajectory jumps in another direction after contacting the constraint surface $\Sigma$ and leaves from another side on $\Sigma$. In this process, the motion state vector $\mathrm{G}$ satisfies the relation as follows:

$G: \Sigma_{i} \rightarrow \Sigma, x^{-}=G x^{+}$,

where $x^{-}$means the position that trajectory contacts the surface before a very short moment, and $x^{+}$means the position that trajectory runs out from the surface. The modulus $G$ is related more to the material and the restitution coefficient.

We conducted an analysis to look for differences in influence of particle materials and plate materials by comparing the normal restitution coefficient of velocity $e_{n}$, which can be defined as follows:

$e_{n}=\left|\frac{v_{o}^{n}}{v_{i}^{n}}\right|$.

In this formula, $v_{i}$ and $v_{o}$ represent the normal components of the approach velocity and the rebound velocity.

Figure 8 shows the restitution coefficient of normal velocity, and each curve represents one kind of particle hitting a plate using a different material (HT250 gray cast iron, 6061 aluminum, and 316 stainless steel). In the upper graph, curves of the normal coefficient of iron particles changing with the impact angle are given. The lower graph represents the normal coefficient of velocity of glass particles with different wall materials at different angles, respectively. The solid lines indicate the wet-surface environmental condition, and the dotted lines indicate the experimental environments were drying.

According to Kantak [10], the normal rebound velocity increased along with an increase in the incident angle when the approach velocity remained unchanged, which meant that the larger the impact angle was, the bigger the normal coefficient of restitution would be when using a large particle impact plate with a thin layer. This conclusion was different from what is shown in Fig. 8. This difference may have been result of different experimental conditions, which meant that the results from the plate with a thin layer were different from the wet surface.

In the study of bounce of large particles, we use $\varepsilon$ to indicate a deformation in the particle [9]. The $\varepsilon$ refers to the elasticity parameter, which describes the deformation of the particle in the elastic process. The elasticity parameter is a ratio of viscous forces and the stiffness of the solid, which means the value of force causes the deformation to divide the value of the parameter that describes how hard it is for the solid to resist deformation. The value of $\varepsilon$ is defined as follows:

Fig. $7 e_{v}$ and $e_{\beta}$ in different impact velocities 
$\varepsilon=\frac{4 K \mu \nu_{0} a^{3 / 2}}{\pi x_{0}^{5 / 2}}$,

where $x_{0}$ is the initial distance from the position where the spheres start to drop to the surface, $v_{0}$ is the incident velocity, $a$ is the particle radius, $\mu$ is the liquid viscosity. The result of $\varepsilon$ in different experimental condition is given in Table 5. The value of $\varepsilon$ refers to the difficulty of resisting deformation - that is, the larger the value, the more easily the deformation occurs.

Table 5 shows obvious differences in the elasticity parameters of using iron particles, whereas the parameters of using glass particles are not differentiated. The parameters in the dry surface condition were all smaller than the parameters in the wet condition, which meant that in the wet surface condition, the particles were more easily deformed.

In Fig. 8, the curves of different plate materials are separated. When iron 45 particles were used for the experiment, under the same experimental conditions, the curve of HT250 gray cast iron plate was higher than the result obtained when using the 316 stainless steel plate, and when the 6061 aluminum alloy wall material was used, the curve was located at the lowest position. The definition of $e_{n}$ is the ratio of the particle speed before and after collision. The bigger restitution coefficient meant less loss of velocity in the headon direction after collision, which meant that less kinetic energy of the particle dissipated in the deformation process. This loss in kinetic energy corresponded to the difficulty of deformation between the particle material and the plate material. A conclusion that can be obtained from Fig. 8 and Table 5 is that HT250 gray cast iron is the most difficult to

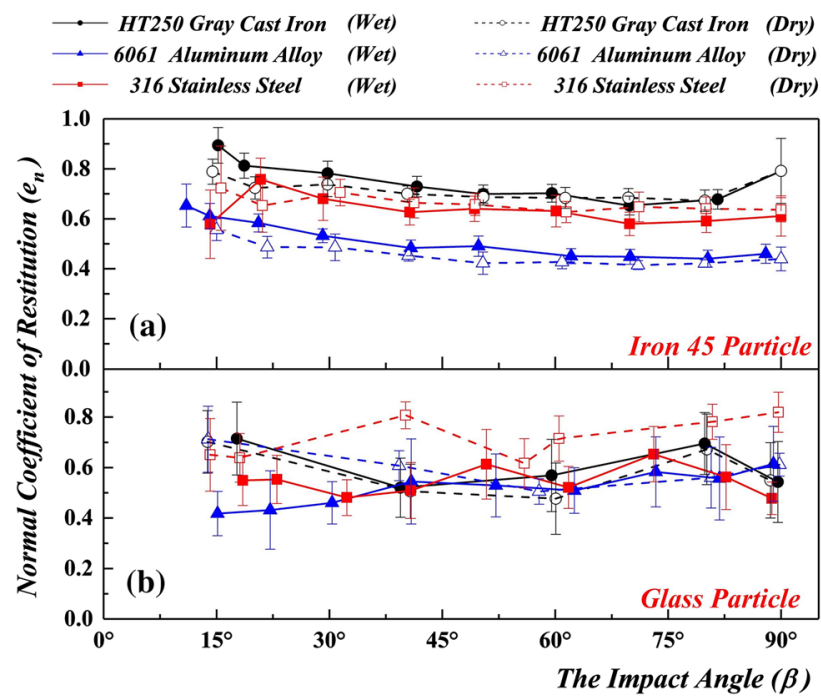

Fig. $8 e_{n}$ in different impact angles: a iron particles; and $\mathbf{b}$ glass particles deform, 6061 aluminum alloy is the most prone to deformation, and 316 stainless steel plate has an intermediate resistance to deformation. This comparison became invalid, however, when using glass particles. In Table 5 , the $\varepsilon$ of iron 45 particles with different plates was much smaller than the $\varepsilon$ of glass particles with different plates. Compared with the $\varepsilon$ of glass particles with different plates, the values of the $\varepsilon$ of iron 45 particles with different plates were much closer to each other, which may have caused the not obvious distribution of $e_{n}$ for glass particles. In addition, the coefficients were smaller in the wet experimental condition than in the dry condition for plastic material particles, and this relationship was opposite when using brittle material particles.

\subsection{The stick-slip bifurcation}

\subsubsection{The influence of the impact angle}

There is a phenomenon called stick-slip bifurcation in the non-smooth dynamic interpretation of the single particle-wall collision model. Stick-slip bifurcation means that the flow in the phase space would stick or slip, which depends mostly on the impact velocity and angle, for a period of time after reaching a certain switching surface before leaving [25].

To study how the impact angle influences the dependence of the stick or the slip, we processed the experimental results further. Based on Hertzian contact theory, numerical simulations of Maw et al. [4] showed that three different types of impacts may occur along with an increase in the impact angle. These three types of impacting motion are full stick, gross slip, and motion between full stick and gross slip. Walton [26] simplified Mas's model, which divided the motion into only stick regime and slip regime.

To discuss this phenomenon, the following elements must be defined [27]:

$\Psi_{\text {in }}=\left|\frac{v_{i}^{t}}{v_{i}^{n}}\right|=\tan \left(90^{\circ}-\beta_{i}\right)$ and $\Psi_{\text {out }}=\frac{v_{o}^{t}}{\left|v_{i}^{n}\right|}=e_{n} \tan \left(90^{\circ}-\beta_{o}\right)$,

where the subscripts $n$ and $t$ denote the normal and tangential component of the impact or rebound velocity, respectively. The tangential coefficients of restitution can be calculated by the following formula:

$e_{t}=\left|\frac{v_{o}^{t}}{v_{i}^{t}}\right|$.

Researchers usually use $e_{t}$ to describe the effect of the friction factor when the collision in the horizon is more like friction. Walton's model makes use of the normal and tangential coefficient of restitution $e_{n}$ and $e_{t}$, and the Coulomb 
coefficient of sliding friction $\mu_{c}$. Walton gave the boundary of whether the impacting particle is sticking on the plate or slipping along with the surface, as follows:
Thornton et al. [30] presented a similar theoretical expression, in which the velocity was raised to a quarter power to determine whether the restitution coefficient was elastic in

$\Psi_{\text {out }}=\left\{\begin{array}{c}-e_{t} \Psi_{\text {in }} \\ \Psi_{\text {in }}-\mu_{c}\left(1+\frac{1}{C}\right)\left(1+e_{n}\right)\end{array} \quad \Psi_{\text {in }} \leq \Psi_{\text {in }}^{*} \Psi_{\text {in }} g t ; \Psi_{\text {in }}^{*} \quad \begin{array}{l}\text { Stick } \\ \text { Slip }\end{array}\right.$ and $\Psi_{\text {in }}^{*}=\frac{7}{2} \mu_{d} \frac{1+e_{n}}{1+e_{t}}$

where $C$ is the radius of gyration normalized by the particle radius, $C=2 / 5$ for a homogeneous solid sphere, and $\mu_{d}$ means the static friction coefficient, based on the continuity of $\psi_{\text {out }}$ at $\psi^{*}{ }_{i n}$. In this study, we applied Walton's model in our experimental data to calculate the $\psi_{\text {in }}$ and the $\psi_{\text {out }}$ of iron particles and glass particles, respectively.

In Fig. 9a, we can see a turning point in the region of $\psi_{\text {in }}$ from 0.5 to 1.5 , which meant there was a critical angle in which to decide whether the particle would stick or slip on the plate after collision in this range. In Fig. 9b, however, the experimental environment was immersed, and we do not find an obvious viscosity-slip boundary because the fluid acts as a lubricant. According to the formula (17), Table 6 gives the boundary impact angle of the wall stick-slip phenomenon of the particles using the two materials (iron 45 and glass) and gives the three materials under the dry wall condition.

\subsubsection{The influence of the impact velocity}

Researchers used to think that the energy of the particle was absorbed by the elastic wave in the course of the collisionthat is, the faster the particle was, the more energy would be absorbed. Reed et al. [28] confirmed that the elastic wave was not the main reason for this phenomenon. Goldsmith et al. [29] observed this phenomenon, too, and proposed that this phenomenon was caused by vibration. perfectly plastic spheres. To explore the causes of this phenomenon, we analyzed impact craters using the ultra-depth of field three-dimensional (3D) microscope. The impact crater morphology and the corresponding 3D scanning image are shown in Fig. 10.

Figure 11 shows the impact crater under different impact velocities at the 50-degree impact angle. The wall material was 6061 aluminum with a dry surface.

The downward trend of $e_{\beta}$ was the result of the fact that the particles were more likely to slip on the surface when the impact speed increased. The work of sliding friction caused part of the energy to be dissipated in the interaction with the wall. Meanwhile, the sliding of the particles caused the plastic deformation of the wall, and the larger impact velocity was, the larger the deformation region was. We concluded that the particle would stick on the plate in the small velocity, but it was more likely to slip on the plate at a higher velocity. The particle also would squeeze the wall and cause the plastic deformation to occur on the plate when it slid.

For this experiment using iron 45 particles and 6061 aluminum alloy plate in the condition of a 50-degree impact angle, we also concluded that the critical velocity for determining the stick-slip motion was between 2.76 and $3.29 \mathrm{~m} / \mathrm{s}$. (a)

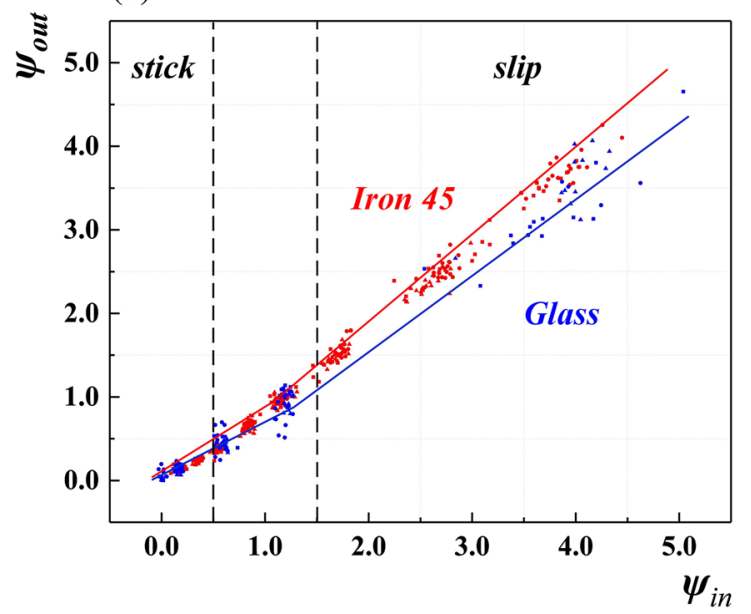

(b)

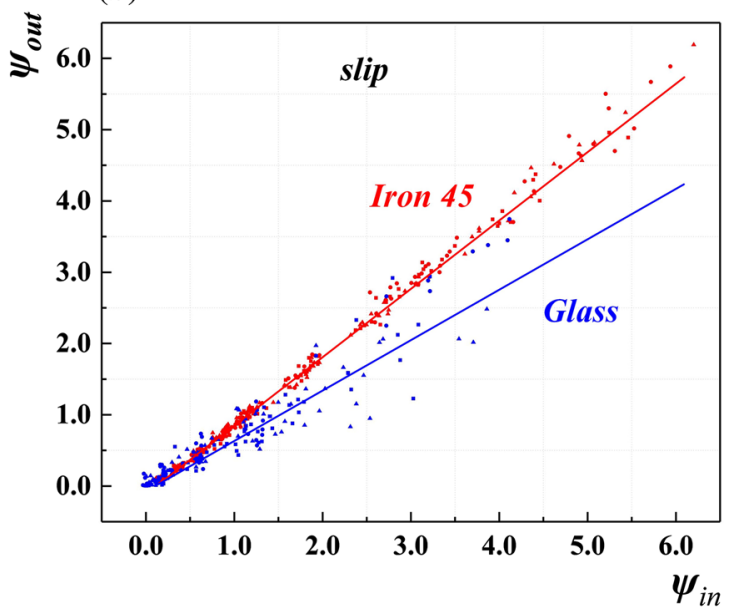

Fig. 9 The collision results fitted with Walton's collision model: a dry experimental condition; and b wet experimental condition 
Table 6 Values of the elasticity parameter in different experimental conditions

\begin{tabular}{llll}
\hline Particle & Plate & $\mu_{d}$ & $\beta_{\Psi}$ \\
\hline Iron 45 & HT250 Gray cast iron & 0.4 & 31.75 \\
& 6061 Aluminum & 0.45 & 38.11 \\
& 316 Stainless steel & 0.5 & 31.07 \\
Glass & HT250 Gray cast iron & 0.5 & 25.36 \\
& 6061 Aluminum & 0.6 & 27.59 \\
& 316 Stainless steel & 0.7 & 24.04 \\
\hline
\end{tabular}

\section{Conclusions}

In this study, we proposed a detailed model for investigation of particle-wall collision on mechanics under the wet and dry conditions and compared our finding with experimental results. We considered several elements that influenced the approach and rebound processes using the iron 45 particles and glass particles, including the impact angle, the plate materials, the experimental environments, and the impact velocities. Guided by the nonlinear dynamics, we mainly discussed the influence of plate materials on the phenomena of stick-slip in particle-wall collision at various impact angles and different impact velocities.

We experimentally obtained the $e_{v}, e_{n}, e_{t}$, and $e_{\beta}$ of plates using three different kinds of materials (i.e., 316 stainless steel, 6061 aluminum alloy, and HT250 gray cast iron) versus particles in two different materials (i.e., 45 iron and glass) under dry and wet conditions. The experimental results are as follows:

- For both the plastic particles represented by the iron 45 particles and the brittle particles represented by the glass,

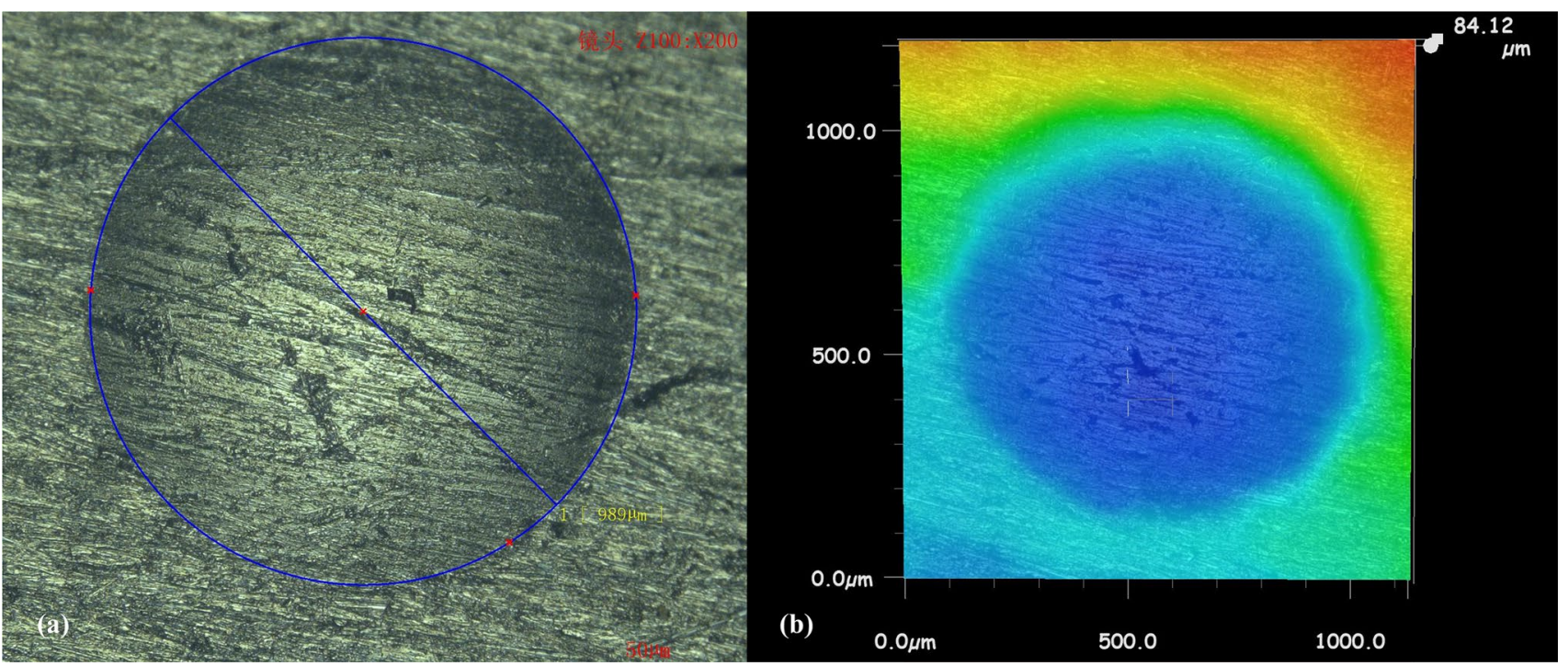

Fig. 10 The particle-wall collision craters shoot by the ultra-depth of field 3D microscope

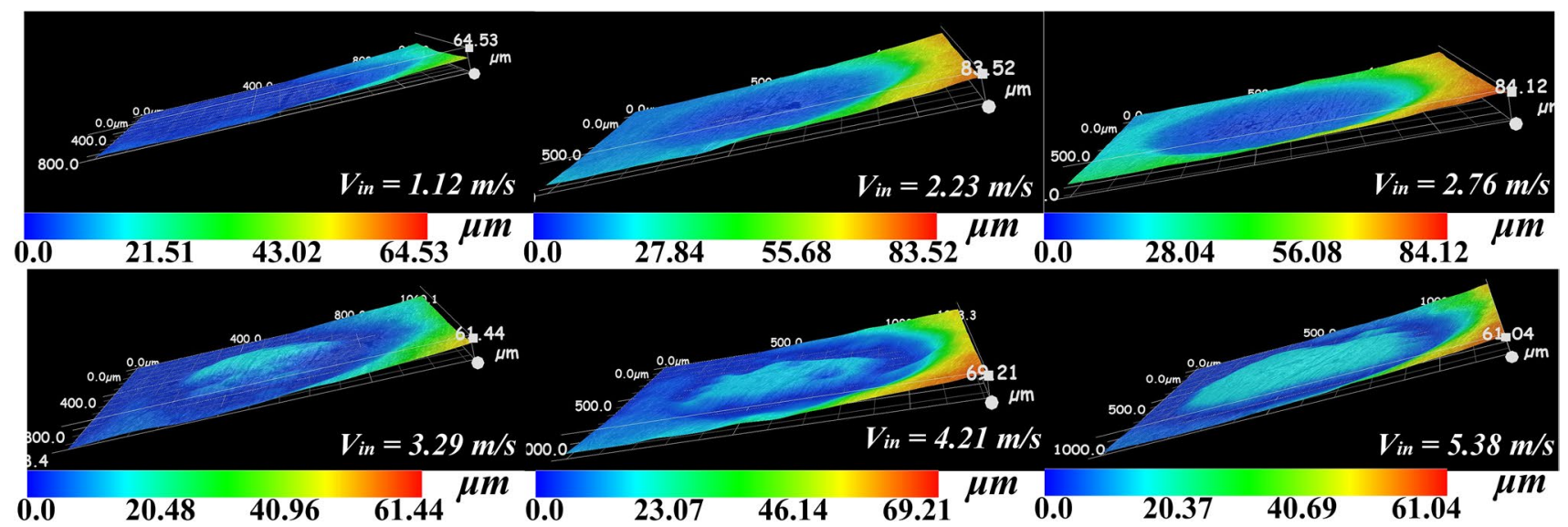

Fig. 11. 3D scanning figure of particle-wall collision craters in different impact velocities 
$e_{\nu}$ decreased along with the increase of the impact angle, and $e_{\beta}$ increased with the increase of the impact angle. Based on this phenomenon, it can be concluded that the restitution coefficients were much more related to the impact angle.

- For the iron 45 particles, the distribution of $e_{v}$ and $e_{\beta}$ were related to the material characteristics of the plates obviously. Although the distribution for the glass particles was much less obvious.

- The experimental results for the restitution coefficients of the iron 45 particles and the glass particles in the wet surface condition were smaller than the results obtained in the dry surface condition for most impact angles. This phenomenon revealed the influence of the viscous liquid.

- The restitution coefficients decreased slightly with an increase in the impact velocity, which indicated the change in the contact state of the particle and the plate with the impact velocity.

- In the normal direction, $e_{n}$ retained nearly the same value no matter what change was made to the impact angle. The distribution of $e_{n}$ was obvious as well.

We then discussed results based on the nonlinear dynamics, which focused mostly on the phenomenon of stick-slip motion and the grazing impact. The single particle-wall collision was seen as a non-smooth dynamical system. The conclusions are as follows:

- The twisting in the curves was caused by the change in the contact state between particles and the plate along with the change of $\beta_{i}$. This phenomenon revealed that the single particle would knock into the plate with two different states-that is, sticking on the plate or slipping along the surface for a little distance before it bounced.

- In this system, the distribution the curves of $e_{v}$ and $e_{n}$ was related to the material characteristics of the particles and plates. The analysis showed that the distribution was significantly related to the elasticity parameter $\varepsilon$, which meant the resistance to deformation. For iron 45 particles, HT250 gray cast iron had the largest $\varepsilon$, and 6061 aluminum alloy had the smallest $\varepsilon$, whereas the $\varepsilon$ of the 316 stainless steel plate was between these two kinds of materials. For glass particles, however, this distinction was not obvious for a similar $\varepsilon$.

We also conducted microscopic study using an ultradepth of field 3D microscope. The conclusions are as follows:

- After photographing the specific surfaces of the plate shot by the particle at different impacting speeds, we observed a phenomenon of thickening in the center of the impact crater when the impact velocity increased, which indicated that the stick-slip motion was related to the impact velocity.

- On the basis of the microtopography, the critical velocity was between 2.76 and $3.29 \mathrm{~m} / \mathrm{s}$ in this experiment.

Acknowledgements We thank LetPub (www.letpub.com) for its linguistic assistance during the preparation of this manuscript.

Author contributions Conceptualization, Langyu Ji and Yi Li; methodology, Xueyu Chen; software, Xueyu Chen; validation, Langyu Ji; formal analysis, Langyu Ji; data curation, Yi Li; writing — original draft preparation, Xueyu Chen; writing - review and editing, Xueyu Chen and $\mathrm{Yi} \mathrm{Li}$; supervision, Yi Li; project administration, Yi Li. All authors have read and agreed to the published version of the manuscript.

Funding This work was supported by the National Natural Science Foundation of China (No. 51976197), Key Research and Development Program of Zhejiang Province (No. 2020C03099), and General Research Program of Department of Education of Zhejiang Province (No. Y201942795).

Open Access This article is licensed under a Creative Commons Attribution 4.0 International License, which permits use, sharing, adaptation, distribution and reproduction in any medium or format, as long as you give appropriate credit to the original author(s) and the source, provide a link to the Creative Commons licence, and indicate if changes were made. The images or other third party material in this article are included in the article's Creative Commons licence, unless indicated otherwise in a credit line to the material. If material is not included in the article's Creative Commons licence and your intended use is not permitted by statutory regulation or exceeds the permitted use, you will need to obtain permission directly from the copyright holder. To view a copy of this licence, visit http://creativecommons.org/licenses/by/4.0/.

\section{References}

1. Govers E, Vertogen G (1985) Erratum: elastic continuum theory of biaxial nematics [Phys. Rev. A 30, 1998 (1984)]. Phys. Rev. A 31(3), 1957-1957. https://doi.org/10.1103/PhysRevA.31.1957.3

2. Johnson KL, Kendall K, Roberts AD (1971) Surface energy and the contact of elastic solids. Proc R Soc Lond A 324(1558):301313. https://doi.org/10.1098/rspa.1971.0141

3. Johnson KL, Kendall K, Roberts AD (1971) Surface energy and the contact of elastic solids. Proc R Soc Lond Ser A Math Phys Sci (1934-1990) 324(1558):301-313. https://doi.org/10.1098/ rspa.1971.0141

4. Maw N, Barber JR, Fawcett JN (1976) The oblique impact of elastic spheres. Wear 38(1):101-114. https://doi.org/10.1016/ 0043-1648(76)90201-5

5. Sommerfeld M, Huber N (1999) Experimental analysis and modelling of particle-wall collisions. Int J Multiphase Flow 25(67):1457-1489. https://doi.org/10.1016/S0301-9322(99)00047-6

6. Grant G, Tabakoff W (1975) Erosion prediction in turbomachinery resulting from environmental solid particles. J Aircraft 12(5):471478. https://doi.org/10.2514/3.59826

7. Newton I (1982) Philosophiae naturalis principia mathematica. Lyon Méd 225(6):553-558. https://doi.org/10.1007/BF01491650

8. Tabakoff W, Malak MF, Hamed A (1987) Laser measurements of solid-particle rebound parameters impacting on 2024 aluminum and 6A1-4V titanium alloys. AIAA J 25(5):721-726. https://doi. org/10.2514/3.9688 
9. Davis RH, Serayssol J-M, Hinch EJ (1986) The elastohydrodynamic collision of two spheres. J Fluid Mech 163:479-497. https://doi.org/10.1017/S0022112086002392

10. Kantak AA (2005) Wet particle collisions. Doctor of Philosophy, University of Colorado Department of Chemical and Biological Engineering

11. Mueller P, Antonyuk S, Stasiak M, Tomas J, Heinrich S (2011) The normal and oblique impact of three types of wet granules. Granular Matter 13(4):455-463. https://doi.org/10.1007/ s10035-011-0256-5

12. Yang F-L, Hunt ML (2006) Dynamics of particle-particle collisions in a viscous liquid. Phys Fluids 18(12):121506. https://doi. org/10.1063/1.2396925

13. Stocchino A, Guala M (2005) Particle-wall collision in shear thinning fluids. Exp Fluids 38(4):476-484. https://doi.org/10.1007/ s00348-005-0928-1

14. Joseph GG, Hunt ML (2004) Oblique particlewall collisions in a liquid. J Fluid Mech 510:71-93. https://doi.org/10.1017/S0022 11200400919X

15. Gollwitzer F, Rehberg I, Kruelle CA, Huang K (2012) Coefficient of restitution for wet particles. Phys Rev E 86(1):011303. https:// doi.org/10.1103/PhysRevE.86.011303

16. Givehchi R, Tan Z (2015) The effect of capillary force on airborne nanoparticle filtration. J Aerosol Sci 83:12-24. https://doi.org/10. 1016/j.jaerosci.2015.02.001

17. Tang T, He Y, Tai T, DEM Wen D (2017) Numerical investigation of wet particle flow behaviors in multiple-spout fluidized beds. Chem Eng Sci 172:79-99. https://doi.org/10.1016/j.ces.2017.06. 025

18. Li X, Dong M, Li S, Shang Y (2019) Experimental and theoretical studies of the relationship between dry and humid normal restitution coefficients. J Aerosol Sci 129:16-27. https://doi.org/10. 1016/j.jaerosci.2018.12.006

19. Sondergaard R, Chaney K, Brennen CE (1990) Measurements of solid spheres bouncing off flat plates. J Appl Mech 57(3):694-699.
20. Gonzalez RC, Woods, et al. (2009) Digital image processing using MATLAB, 2nd ed. Pub. House of Electronics Industry. https://doi. org/10.9774/GLEAF.978-1-909493-38-4_2

21. Image Process Toolbox User's Guide. Mathworks Inc, 2020. https://ww2.mathworks.cn/help/images/index.html.

22. Johnson KL (1985) Contact mechanics. Cambridge University Press, Cambridge

23. Moreau JJ, Panagiotopoulos PD (eds) (1988) Nonsmooth mechanics and applications. Springer, Vienna. https://doi.org/10.1007/ 978-3-7091-2624-0

24. Nordmark AB (1991) Non-periodic motion caused by grazing incidence in an impact oscillator. J Sound Vib 145(3):279-297. https://doi.org/10.1016/0022-460X(91)90592-8

25. Nusse HE, Ott E, Yorke JA (1994) Border-collision bifurcations: an explanation for observed bifurcation phenomena. Phys Rev E 49(2):1073-1076. https://doi.org/10.1103/PhysRevE.49.1073

26. Walton OR, Roco MC (1993) Numerical simulation of inelastic, frictional particle-particle interactions. In: Particulate two-phase flow; Butterworth-Heinemann, pp 884-907

27. Drost LJ (2015) Oblique particle-wall collisions in a viscous fluid. master thesis, Delft University of Technology

28. Reed J (1985) Energy losses due to elastic wave propagation during an elastic impact. J Phys D Appl Phys 18(12):2329-2337. https://doi.org/10.1088/0022-3727/18/12/004

29. Goldsmith W, Frasier JT (1961) Impact: the theory and physical behavior of colliding solids. J Appl Mech 28(4):639-639. https:// doi.org/10.1115/1.3641808

30. Thornton C, Ning Z (1998) A theoretical model for the stick/ bounce behaviour of adhesive elastic-plastic spheres. Powder Technol 99:154-162. https://doi.org/10.1016/S0032-5910(98) 00099-0

Publisher's Note Springer Nature remains neutral with regard to jurisdictional claims in published maps and institutional affiliations. 